\title{
SKETCH BASED REDUCED MEMORY HOUGH TRANSFORM
}

\author{
Levi Offen \& Michael Werman
}

\author{
Computer Science \\ The Hebrew University of Jerusalem \\ Jerusalem, Israel
}

\begin{abstract}
This paper proposes using sketch algorithms to represent the votes in Hough transforms. Replacing the accumulator array with a sketch (Sketch Hough Transform - SHT) significantly reduces the memory needed to compute a Hough transform. We also present a new sketch, Count Median Update, which works better than known sketch methods for replacing the accumulator array in the Hough Transform.
\end{abstract}

Index Terms - Image processing, Sketch, Line detection, Hough transform, Random mapping, Memory Saving

\section{INTRODUCTION}

Extracting shapes from images is a key issue in vision and image processing. Object detection, especially line detection, is a fundamental operation used in a wide range of applications.

The Hough transform [1], HT, and the Generalized Hough Transform [2], GHT, are tools based on a voting scheme where image elements vote for parameters of the geometric object. Unfortunately, these methods have large memory and computation time requirements as the parameter space increases exponentially with the dimension of the problem, the number of parameters. On the other hand, reducing the image or the parameter space by quantization significantly lowers accuracy.

Sketches as methods to approximate frequencies have been successfully used in big data and streaming, where massive data needs to be processed in memory and time efficient manner [3, 4, 5]. Sketch algorithms refer to a class of streaming algorithms that represent a large dataset with a compact summary, typically much smaller than the full size of the input.

One of the problems solved using sketches is the 'frequent items' problem. Given an sequence of items, find all items whose frequency ('vote value') exceeds a specified fraction of the total number of items: A wide variety of algorithms and heuristics have been proposed for this problem, based on sampling, hashing, and counting (see [6, 7] for surveys).

This research was supported by the Israel Science Foundation and the Israel Ministry of Science and Technology.

\subsection{Our Contribution}
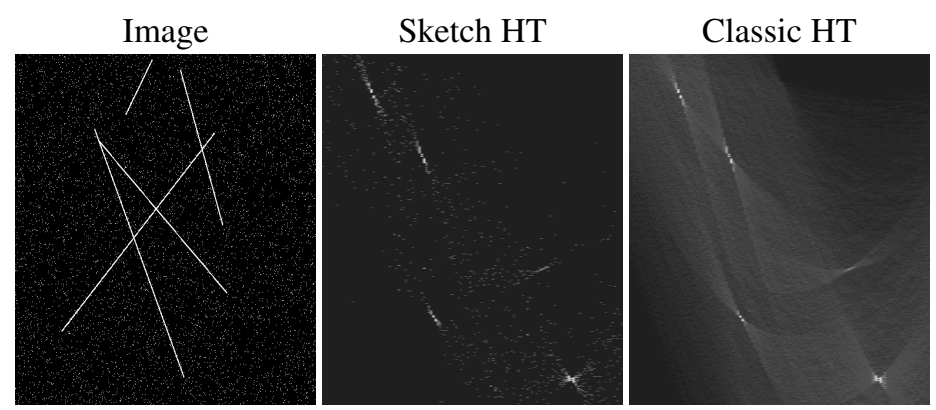

Fig. 1: The motivation for SHT. Comparing the votes (in parameter space $O$ ) of the Classic HT to the 'top-frequentvotes' on Sketches HT. It can be seen that many small votes on the right image are omitted on the middle image, while votes around the peaks still preserved.

Hough transform algorithms detect objects by searching for a local peak in the object parameter space $O$, by the following major steps: 1 . Image elements, $e$, vote for the cells in $O$ that agree with e. 2. Local maxima, peaks, in the accumulator, are the detected shapes where the votes are stored in an array with the dimension of $O$. We propose replacing the accumulator array with a much smaller sketch.

The 'frequent items' (or 'heavy hitters') problem is not exactly what is needed for the 'peak detection' in Hough transforms, for several reasons:

- Due to geometric quantization and noise there are several points related to an object and we only want one.

- We want to recognize the objects in the image and are not usually interested in their exact number of votes.

- There is often a significant amount of noise in the image, which should be ignored.

In this paper we show how a sketch algorithms for the 'frequent items' problem can improve Hough transform algorithms, using much less memory and with a better robustness to noise. The main idea is that the votes are only approximated and the peak detection is carried out only around the 

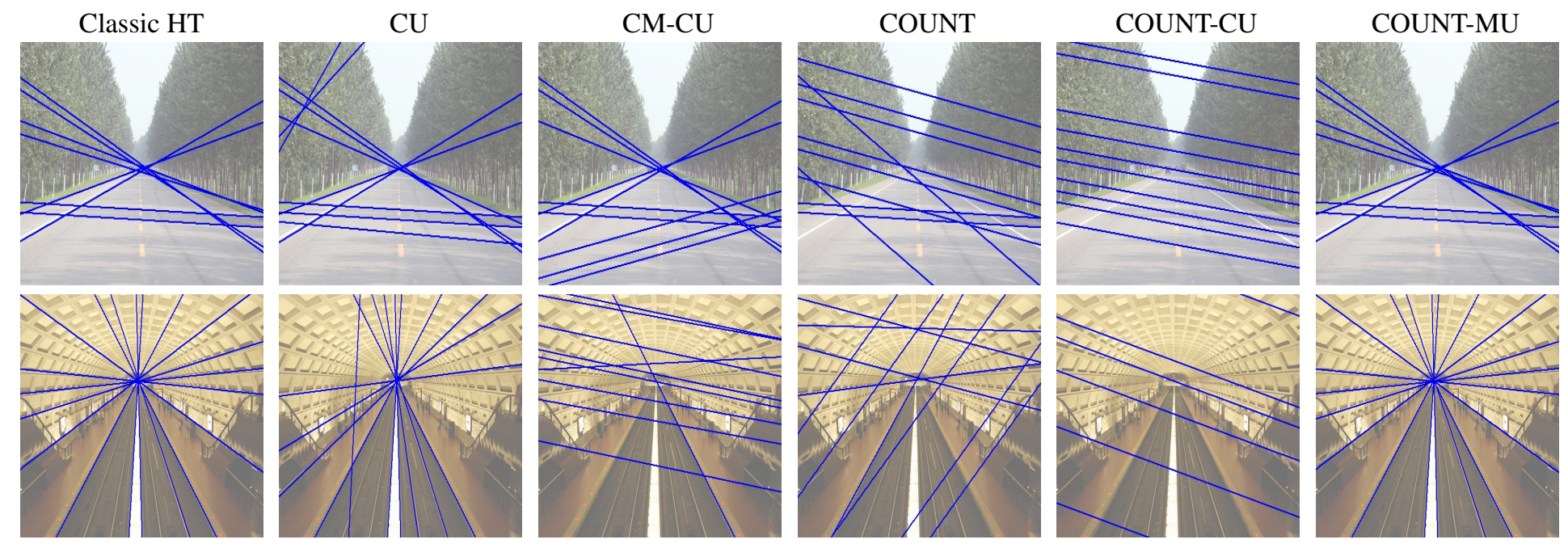

Fig. 2: Detected lines in an image. Classic Hough Transform and various Sketch Hough Transforms.

'frequent votes'. Figure 1 shows the difference between the full object parameter space accumulator in Classic HT and the 'top-frequent-votes' of the Sketch HT.

We also propose a new sketch algorithm, Count Median Update, which improves the estimation accuracy compared to known methods.

\subsection{Previous Work}

\subsubsection{Hough Transform Algorithms}

The main disadvantages of HT are long computation time and large data storage. Many implementations have being proposed to alleviate these issues [8].

There are probabilistic methods to speed up the HT such as Probabilistic HT [9], PHT, and Randomized HT [10, 11, [12], RHT. Although RHT and PHT are computationally fast, they are sensitive to noise and occlusions, since the noise pixels have extra impact on these randomized algorithms [13, 14]. These algorithms use randomness for choosing points in the image space, while we suggest using randomness in parameter space. Our algorithms can also be combined with the previous random methods.

\subsubsection{Data Streaming Sketch Algorithms}

Sketches are concise data summaries of a high-dimensional vector which can be used to estimate queries on it. The sketch is a linear projection of the input vector with random vectors defined by hash functions. Increasing the range of the hash functions co-domain $(w)$ increases the accuracy of the estimation, and increasing the number of hash functions decreases the probability of a bad estimate.

The sketch is a $d \times w$ array $C$, and supports INCREMENT and $Q U E R Y$, which can be used for solving the 'frequent items' problem. We outline two sketching approaches

- Count sketch [3] (COUNT) (aka AMS[5]) - There are 2 hash functions per row; $h_{i}(x)$ and $s_{i}(x)$ mapping input items onto $[w]$ and $\{+1,-1\}$ respectively.

$$
\begin{aligned}
& \text { INCREMENT(x) : } \\
& \underset{1 \leq i \leq d}{\forall} C\left[i, h_{i}(x)\right] \leftarrow C\left[i, h_{i}(x)\right]+s_{i}(x) \\
& \text { QUERY }(x) \text { : } \\
& \underset{1 \leq i \leq d}{\operatorname{median}} C\left[i, h_{i}(x)\right] * s_{i}(x)
\end{aligned}
$$

- Count Min sketch[4] (CM) - the sketch is similar to COUNT but without the sign hash function. In contrast to COUNT this algorithm returns a biased estimator, overestimating the count.

$$
\begin{aligned}
& \text { INCREMENT(x) : } \\
& \underset{1 \leq i \leq d}{\forall} C\left[i, h_{i}(x)\right] \leftarrow C\left[i, h_{i}(x)\right]+1 \\
& \text { QUERY }(x) \text { : } \\
& \min _{1 \leq i \leq d} C\left[i, h_{i}(x)\right]
\end{aligned}
$$

Let $P$ be the number of items inserted in the sketch, $f_{i}$ the number of times element $i$ was inserted in the sketch ( $\left.\sum_{i} f_{i}=P\right)$, and using $d$ hash functions ( $d \times w$ memory), sketches guarantee:
Sketch type
Estimation Accuracy
COUNT $(x)$
$O\left(\frac{1}{\sqrt{w}} \sqrt{\sum_{i}\left(f_{i}\right)^{2}}\right)$
$C M(x)$
$O\left(\frac{1}{w} P\right)$
$1-\frac{4}{e^{d}}$
$1-\frac{1}{e^{d}}$

Success Probability

Estimation accuracy is a bound on the distance of sketch 
$Q U E R Y(x)$ from the real vote value of $x, f_{x}$, and success probability is the probability that this bound fulfilled.

Methods used to improve sketches include:

- Conservative Update (CU) - conservative updates [15, [16] were extended to sketches [6, 17] to avoid unnecessary updates and reduce overestimation. $C\left[i, h_{i}(x)\right]$ is incremented only if ${ }^{1} C\left[i, h_{i}(x)\right] \leq Q U E R Y(x)$. CU depends on the order of the incremented items and although it does not guarantee improvement it often does.

- Lossy counting (L) - lossy counting [18] was extended to sketches [19, 17] by removing small votes. In this approach, the input is divided into $k$ parts. After processing the $t^{\prime}$ th part, small cells $0<|C[i, j]| \leq t$ (or $\sqrt{t}$ ), are reduced. In our experiments on images lossy counting did not improve the results so we do not mention this method again.

Sketches solve the top-k frequent items problem by maintaining a top-k list which is updated during the INCREMENT $(x)$ [20] or by comparing the $Q U E R Y(x)$ results for all the $x$ 's in parameter space.

\section{NEW COUNT SKETCH}

While CM with CU shows significant improvement over CM in many cases, [19] show that CM-CU can reduce the overestimation error by at least 1.5 , it appears that COUNT-CU [17] gives little improvement. As CU reduces only over-estimate errors and COUNT also contains under-estimate errors.

We propose a new variant of conservative update - Count Median Update (COUNT-MU) - that reduces both over and under estimate errors.

$$
\begin{aligned}
& \text { INCREMENT(x): } \\
& \text { median } \leftarrow \underset{1 \leq i \leq d}{\operatorname{median}} \quad C\left[i, h_{i}(x)\right] * s_{i}(x) \\
& \underset{1 \leq i \leq d}{\forall} \quad \text { If } \quad \text { median }==C\left[i, h_{i}(x)\right] * s_{i}(x): \\
& C\left[i, h_{i}(x)\right] \leftarrow C\left[i, h_{i}(x)\right]+s_{i}(x)
\end{aligned}
$$

The motivation for this method is similar to CM-CU, updating $x$ should only affect the cells equal to $Q U E R Y(x){ }^{2}$ The other counters are 'wrong' since they were notably influenced by other elements, so incrementing them will increase noise and inaccuracy.

Our new sketch algorithm is significantly better than COUNT-CM and usually better than CM-CU (see 4) and could possibly be of use in other streaming data/NLP queries.

\footnotetext{
${ }^{1} C\left[i, h_{i}(x)\right] * s_{i}(x)$ for COUNT

${ }^{2}$ updating in the range [median_low, median_high] for even $d$ or $[$ median -1, median +1$]$ for odd $d$, instead of just median slightly improves results
}

\section{SKETCH HOUGH TRANSFORM (SHT)}

We claim that any algorithm which estimates the 'frequent items' can be used to improve Hough transforms algorithms.

The Hough Transform's parameters are the polar coordinates of the line, $\theta \in[0, \pi]$ and $\rho \in R$, which are the angle of the normal to the line and the distance from the origin to the line. Let $s k t$ be the used sketch, $d$ the number of hash functions used in skt, mem the memory used by skt (the size of hash's co-domain $\times d$ ), peaks_num the maximum number of lines we expect to find in the image, $\rho_{-} \max$ the maximum distance of a line in the image from the origin, and $|P|$ the number of edge points in the image.

The Classic Hough Transform, CHT, stores the line votes in an accumulator array which ranges over all the object space - \# of angles $\times 2 \rho \_$max. Algorithm 1 . SHT, replaces this accumulator with a smaller sketch, using mem memory and with a $\Theta\left(1-\frac{1}{e^{d}}\right)$ probability returns a superset of the CHT result.

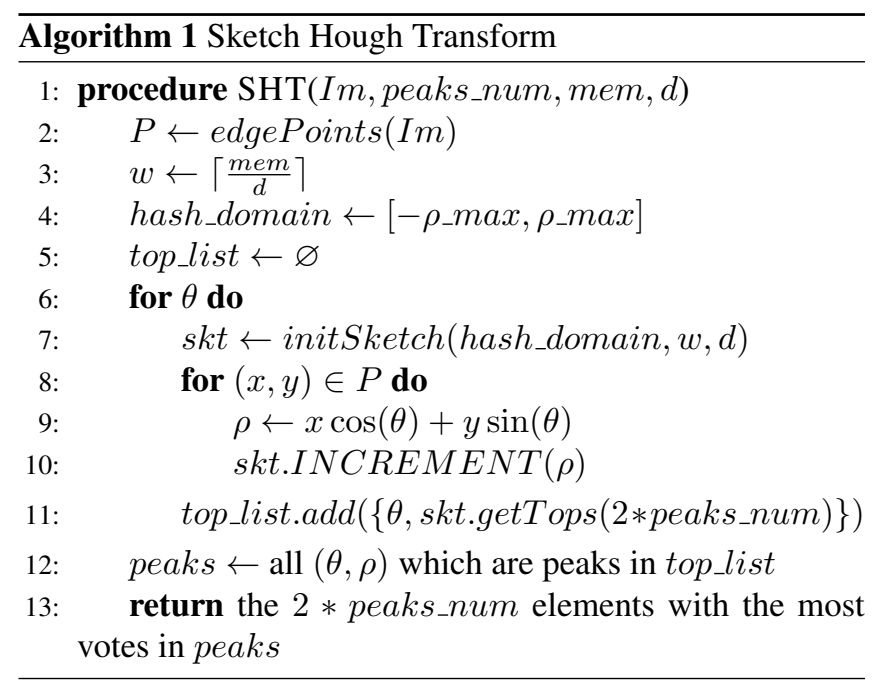

initSketch(hash_domain, $w, d$ ) returns a sketch which stores votes for elements from hash_domain using $d$ hash functions to a size $w$ co-domain - using $w \times d$ memory. getTops $(k)$ returns the top- $k$ 'frequent items' from the sketch and is calculated at line 10 with $O$ (peaks_num) memory.

top_list size can be limited to $O$ (peaks_num) by searching locally for peaks within windows of a fixed number of angles.

Although the number of votes for a line in SHT is different (depending on $|P|$, mem, $d$ and $s k t$ ) from the number of votes in CHT, using the right configuration for mem, $d$ (see 4.1) results in a superset of lines in almost the same order (sorted by votes) as CHT. Additionally, a simple check can remove false lines which do not exist in the image. 


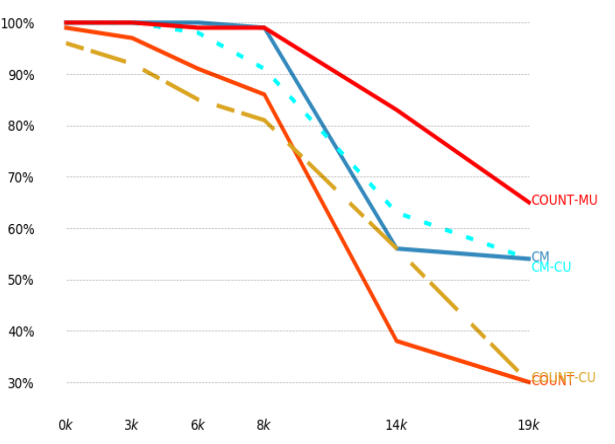

(a)

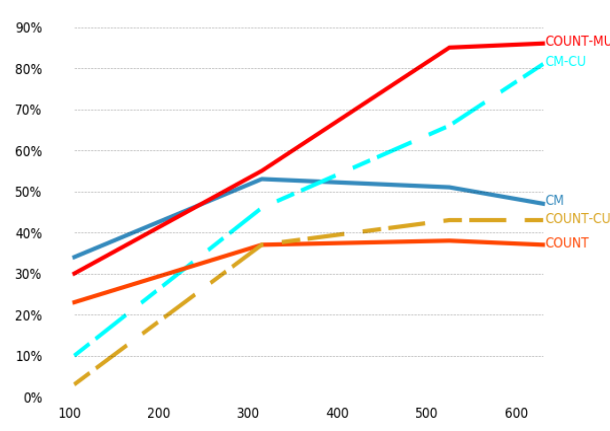

(b)

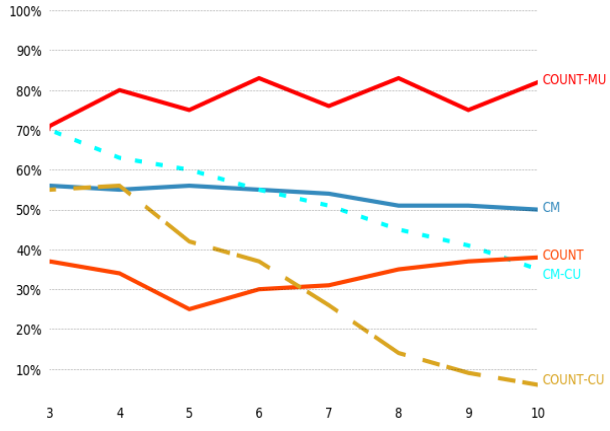

(c)

Fig. 3: (a) Quality as a function of of noise in 1000's of edge elements.

(b) Quality as a function of memory.

(c) Quality as a function of number of hash functions.

\section{EXPERIMENTS}

In order to show the effectiveness of the proposed Sketch Hough Transform (SHT) we run it with several sketching methods: Count Min (CM), Count Min with conservative update (CM-CU), Count (COUNT), Count with conservative update (COUNT-CU), and Count Median Update (COUNT$\mathrm{MU}$ ), our method. All of them with and without lossy counting.

The accuracy of SHT results were calculated by comparing them to the result of CHT using an accumulator of $180 \times 1024$ (184320) memory. Each algorithm was run 10 times on an image and the results quality (mean accuracy) is reported.

\subsection{Synthetic Line Images}

We created 204 synthetic images $(512 \times 512)$ containing 1 5 random lines and added uniform noise. We ran SHT algorithms 10 times on each image, and the quality of the results were compared to the result of the classic Hough Transform. As lossy counting did not have a significant effect on the sketches in these cases, we don't show lossy counting results.

Plot 3 (a) shows the dependence of SHT quality on the amount of noise for 275 bytes of memory. It can be clearly seen that the results of COUNT-MU are superior to all the other sketches. The advantage of our method increases with the noise.

Plot 3 (b) shows the dependence of SHT quality on sketch memory size (hash co-domain $\times$ number of hash functions) for images with $19 \mathrm{k}$ noise points. It can be seen that CM has better results than $\mathrm{CM}-\mathrm{CU}$ for sketches with small memory, while CM-CU is better with memory size above 420 . Our method, COUNT-MU, is superior to all other methods.

Plot 3 (c) shows the dependence of SHT quality on the number of hash functions, using 210 bytes of memory. The seesaw pattern in COUNT-MU is a result of the difference in the definition of median for an even or odd number of elements, number of hash functions.

\subsection{Real Images}

We ran the SHT on 15 random real images of various sizes containing roads, train tracks, skylines and landscapes. Figure 2 shows the detected lines on an image for Classic HT and SHT with various sketch types.

Table 1: SHT accuracy using 275 bytes of memory is:

\begin{tabular}{|c|c|}
\hline Sketch & Quality \\
\hline \hline CM & $76 \%$ \\
CM-CU & $90 \%$ \\
COUNT & $56 \%$ \\
COUNT-CU & $20 \%$ \\
COUNT-MU & $\mathbf{9 6 \%}$ \\
\hline
\end{tabular}

It can be clearly seen that the results of COUNT-MU, our method, are superior to all other sketches on real images too.

\section{CONCLUSION}

We introduced the Sketch Hough Transform, SHT, algorithm that reduced the amount of memory and increased the robustness to noise compared to the Classic Hough Transform. We showed that the results of SHT, using a small memory are almost the same as the classic Hough Transform.

We also proposed a new sketch, Count Median Update, and showed that this new sketch is significantly superior to other sketching methods especially as the noise in the image increased. 


\section{REFERENCES}

[1] P.V.C. and Hough, "A method and means for recognizing complex patterns," U.S. Patent 3,069,654, December 1962.

[2] D. H. Ballard, "Generalizing the Hough transform to detect arbitrary shapes," Pattern Recognition, vol. 13, no. 2, pp. 111-122, Jan. 1981.

[3] Moses Charikar, Kevin Chen, and Martin FarachColton, "Finding frequent items in data streams," Theoretical Computer Science, vol. 312, no. 1, pp. 3-15, Jan. 2004.

[4] Graham Cormode and S. Muthukrishnan, "An improved data stream summary: the count-min sketch and its applications," Journal of Algorithms, vol. 55, no. 1, pp. 58-75, Apr. 2005.

[5] Noga Alon, Yossi Matias, and Mario Szegedy, "The Space Complexity of Approximating the Frequency Moments," Journal of Computer and System Sciences, vol. 58, no. 1, pp. 137-147, Feb. 1999.

[6] Graham Cormode and Marios Hadjieleftheriou, "Finding the frequent items in streams of data," Communications of the ACM, vol. 52, no. 10, pp. 97, Oct. 2009.

[7] Hongyan Liu, Yuan Lin, and Jiawei Han, "Methods for mining frequent items in data streams: an overview," Knowledge and Information Systems, vol. 26, no. 1, pp. 1-30, Jan. 2011.

[8] Priyanka Mukhopadhyay and Bidyut B. Chaudhuri, "A survey of Hough Transform," Pattern Recognition, vol. 48, no. 3, pp. 993-1010, Mar. 2015.

[9] N. Kiryati, Y. Eldar, and A. M. Bruckstein, "A probabilistic Hough transform," Pattern Recognition, vol. 24, no. 4, pp. 303-316, Jan. 1991.

[10] L. Xu and E. Oja, "Randomized Hough Transform (RHT): Basic Mechanisms, Algorithms, and Computational Complexities," CVGIP: Image Understanding, vol. 57, no. 2, pp. 131-154, Mar. 1993.

[11] S. y Guo, X. f Zhang, and F. Zhang, "Adaptive Randomized Hough Transform for Circle Detection using Moving Window," in 2006 International Conference on Machine Learning and Cybernetics, Aug. 2006, pp. 3880-3885.

[12] J.R. Bergen and (Schweitzer) Shvaytser, "A probabilistic algorithm for computing Hough transforms," Journal of Algorithms, vol. 12, no. 4, pp. 639-656, 1991.
[13] Wei Lu and Jinglu Tan, "Detection of incomplete ellipse in images with strong noise by iterative randomized Hough transform (IRHT)," Pattern Recognition, vol. 41, no. 4, pp. 1268-1279, Apr. 2008.

[14] Si-Yu Guo, Ya-Guang Kong, Qiu Tang, and Fan Zhang, "Probabilistic Hough transform for line detection utilizing surround suppression," in 2008 International Conference on Machine Learning and Cybernetics, July 2008, vol. 5, pp. 2993-2998.

[15] Cristian Estan and George Varghese, "New Directions in Traffic Measurement and Accounting," New York, NY, USA, 2002, SIGCOMM '02, pp. 323-336, ACM.

[16] Saar Cohen and Yossi Matias, "Spectral Bloom Filters," New York, NY, USA, 2003, SIGMOD '03, pp. 241-252, ACM.

[17] Amit Goyal, Hal Daum, III, and Graham Cormode, "Sketch Algorithms for Estimating Point Queries in NLP,' Stroudsburg, PA, USA, 2012, EMNLP-CoNLL '12, pp. 1093-1103, Association for Computational Linguistics.

[18] Gurmeet Singh Manku and Rajeev Motwani, "Approximate Frequency Counts over Data Streams," Hong Kong, China, 2002, VLDB '02, pp. 346-357, VLDB Endowment.

[19] Hal Daum Amit Goyal, "Approximate Scalable Bounded Space Sketch for Large Data NLP,” 2011.

[20] Moses Charikar, Kevin Chen, and Martin FarachColton, "Finding Frequent Items in Data Streams," July 2002, Lecture Notes in Computer Science, pp. 693-703, Springer, Berlin, Heidelberg. 